\title{
Characterization of Oleophobic Functional Surfaces Fabricated by Thermal Imprinting Process
}

\author{
Kwang-Jin Bae, Wenhui Yao, Young-Rae Cho \\ Department of Materials Science and Engineering, Pusan National University, \\ Busan, Korea \\ bae@pusan.ac.kr; yaowh2012@126.com; yescho@pusan.ac.kr
}

\section{Extended Abstract}

Nanostructure is the prerequisite to obtain an appropriate surface roughness for the superhydrophobicity or oleophobicity. Synthetic surfaces with nano-sized bumps have been recently developed based on low-energy surface and multiscale roughness by various nanotechniques [1]. Especially, antifouling, deicing, antibacterial, and self-cleaning surfaces are important for improving the energy efficiency of building, automobile, medical devices, and household care [2]. During the past two decades, superhydrophobic nanostructures and nanocoatings that are inspired by the lotus-leafs effect have been extensively studied. However, studies on the oloephobic surfaces were paid less attention. Therefore, it is a challenge to create functional surfaces which completely resist wetting not only by water, but also by organic liquids such as oils [3].

In this work, a study on fabrication and characterization of oleophobic (repellent to oil) surface using thermal imprint lithography is conducted. For thermal imprinting process, a nickel (Ni) stamp with pillar-array was fabricated. During the imprint lithography, the PMMA (polymethyl methacrylate) substrate was prepared and heated above the glass transition temperature. As a result, the micro-patterned PMMA sheet was successfully formed [Fig. 1]. By application of fluoride coating (DURASURF, HARVES. Co.) and polymer nano-particles stacking on the micro-patterned PMMA sheet, a surface modification was carried out. Thus, the hierarchical complex surfaces which have superhydrophobic and oleophobic properties with complex nano-particles on micro-patterns were created.

This hierarchical structure played an important role in oil-repellent properties. As a result, the imprinted surface from nickel stamp showed contact angle around $150^{\circ}$ for water and $118^{\circ}$ for hexadecane. This method can be applicable for a variety of applications such as self-cleaning, antifouling, and antifrosting. Furthermore, we will discuss the mechanism of creating an oleophobic coating in details.
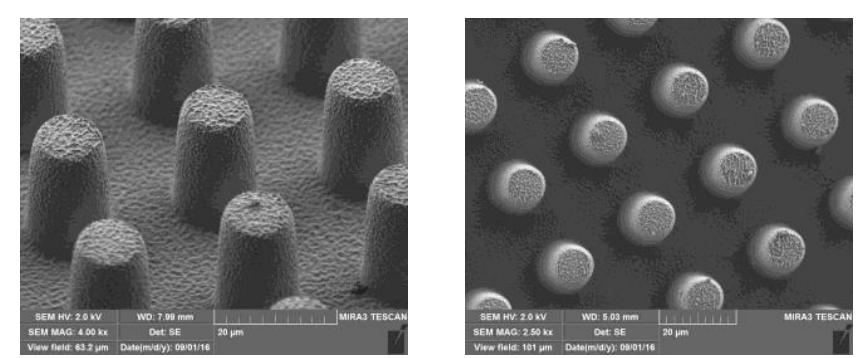

Fig. 1: Micro-patterned bump array using PMMA by thermal imprinting process.

\section{References}

[1] Y. Wang, Y. Shi, L. Pan, L. Peng, S. Zong, Y. Shi and G. Yu, "Multifunctional superhydrophobic surfaces templated from innately microstructured hydrogel matrix," Nano Lett., vol. 14, pp. 4803-4809, 2014.

[2] P. Kim, M. J. Kreder, J. Alvarenga, J. Aizenberg, "Hierarchical or not? Effect of the length scale and hierarchy of the surface roughness on omniphobicity of lubricant-infused substrates," Nano Lett., 13, pp. 1793-1799, 2013. 
[3] T. Li, M. Paliy, X. Wang, B. Kobe, W.M. Lau and J. Yang, "Facile one-step photolithographic method for engineering hierarchically nano/microstructured transparent superamphiphobic surface," ACS Appl. Mater. Interfaces, vol. 7, pp. 10988-10992, 2015. 